\title{
Defibrillator Discharge by ECG Finding
}

National Cancer Institute

\section{Source}

National Cancer Institute. Defibrillator Discharge by ECG Finding. NCI Thesaurus. Code C120609.

An electrocardiographic tracing that is generated by cardioversion or defibrillation. 